\title{
Enthalpy and Volume Changes in Lipid Membranes. I. The Proportionality of Heat and Volume Changes in the Lipid Melting Transition and Its Implication for the Elastic Constants
}

\author{
Holger Ebel, ${ }^{\dagger}$ Peter Grabitz, and Thomas Heimburg* \\ Membrane Thermodynamics Group, Max-Planck-Institute for Biophysical Chemistry, \\ 37077 Göttingen, Germany
}

Received: February 8, 2001; In Final Form: May 3, 2001

\begin{abstract}
Differential scanning calorimetry, pressure calorimetry, and densitometry have been employed to study the relation between volume and enthalpy changes in the melting regime of lipid membranes. We demonstrate a rigid proportional relation between volume expansion coefficient and heat capacity. This result is first shown in densitometric experiments. It implies that calorimetric profiles obey a simple scaling law for the temperature axes in experiments with applied hydrostatic pressure. In a theoretical paper (Heimburg, T. Biochim. Biophys. Acta 1998, 1415, 147-162), we have argued that this relation has far-reaching consequences for the predictiblity of elastic constants from the heat capacity. The proportionality constant between volume and enthalpy changes is found to be independent of the lipid, which has interesting consequences for the calculation of the elastic constants of biological lipid mixtures with unknown composition. We demonstrate this for lung surfactant, which displays a similar relation between volume and enthalpy changes.
\end{abstract}

\section{Introduction}

Lipid membranes undergo a melting transition, which is accompanied by changes in enthalpy ${ }^{1}(20-40 \mathrm{~kJ} / \mathrm{mol})$, volume ${ }^{2}$ (about 4\%), and area (about 25\%). ${ }^{3,4}$ This paper is mainly focused on the changes in lipid volume during a chain melting transition. The volumetric properties of lipids have been extensively studied by many methods, for example by densitometry or dilatometry ${ }^{2,5-12}$ and by investigation of the effect of pressure on light scattering or transmission, ${ }^{13-15}$ spectral changes, ${ }^{16-18}$ heat capacities, ${ }^{19} \mathrm{X}$-ray or neutron scattering, ${ }^{20-23}$ and other methods. Anthony et al. ${ }^{10}$ found that the volume expansion coefficient of single lipid membranes has a very similar temperature dependence as the heat capacity. On the basis of their and our own densitometric experiments, we have argued that this may lead to a simple relation between heat capacity and isothermal volume compressibility. ${ }^{24}$ According to a well-known statistical thermodynamics theorem, the heat capacity is proportional to enthalpy fluctuations, whereas the compressibility is proportional to volume fluctuations. Thus, if enthalpy and volume are closely related, enthalpy and volume fluctuations supposedly are also related functions of the temperature. Therefore, heat capacity and isothermal compressibility are coupled functions. The adiabatic volume compressibility, which is related to the isothermal compressibility via the Maxwell relations, has been monitored in ultrasonic velocity measurements. ${ }^{25}$ Ultrasonic velocity profiles of lipid dispersions display a minimum close to the chain melting transition. It has been shown that the ultrasonic velocity profiles of pure DMPC

* Author to whom correspondence should be addressed. E-mail: theimbu@ gwdg.de, WWW: www.gwdg.de/theimbu.

$\dagger$ Present address: Department of Theoretical and Astrophysics, University of Kiel, 24098 Kiel, Germany.

Abbreviations: DLPC: 1,2-dilauroyl-3-sn-phosphocholine; DMPC: 1,2-dimyristoyl-3-sn-phosphocholine; DPPC: 1,2-dipalmitoyl-3-sn-phosphocholine; LUV: large unilamellar vesicles; MLV: multi-lamellar vesicles. and DMPC-cholesterol mixtures can correctly be predicted from the heat capacity, ${ }^{26}$ if a proportional relationship between heat capacity and isothermal compressibility is assumed.

Furthermore, it has been suggested that a similar close relationship may also exist between lipid area and enthalpy. ${ }^{24}$ From this, a simple coupling between heat capacity and curvature elasticity has been predicted which was confirmed by Dimova et al. ${ }^{27}$ Bending rigidity profiles predicted from the heat capacity are surprisingly similar to experimental results from optical tweezer measurements. ${ }^{27,28}$ Thus it has to be concluded that heat capacity and elastic constants are generally closely related. This may be of great importance in many artificial and biological systems. Vesicle geometries depend on bending and Gaussian curvature moduli. Since the elastic constants change close to the melting transition, structural transitions of vesicles are more likely close to transitions. This effect has been suggested to be the cause for the lipid pretransition $^{31}$ and morphological transitions in certain anionic lipids, ${ }^{29}$ where, close to chain melting, the vesicular geometry reversibly changes into a continuous bilayer network of different mean curvature.

Finally, if heat capacity and membrane bending elasticity are coupled, changes in curvature will also affect melting profiles. ${ }^{29,30}$

This paper is dedicated to the detailed exploration of the relationship between volume and enthalpy. We employ differential densitometry and pressure calorimetry. In contrast to previous densitometric measurements we are not primarily interested in the absolute volume changes between two different phases but in the detailed temperature dependence of enthalpy and volume in the whole melting regime. We demonstrate that pressure calorimetry is suitable to investigate the temperature dependence of the lipid volume since it has a very precise control over the absolute temperature stability. The interesting consequences of our findings are discussed. 


\section{Materials and Methods}

Phospholipids were purchased from Avanti Polar Lipids (Birmingham, AL) and used without further purification. Heat capacities were recorded on a VP-calorimeter from Microcal, Inc. (Northampton, MA) at scan rates of $5 \%$ h and $0.2^{\circ} / \mathrm{h}$ for the very cooperative transitions of multilamellar vesicles. Pressure calorimetry was performed on this instrument using a self-built pressure capillary. The pressure was controlled with nitrogen gas and measured with a sensor (EBM 6045) by Nova Swiss (Effretikon, Switzerland). During DSC scans the pressure was maintained with an error of less than $0.5 \%$ which displayed a slight systematic temperature dependence. Since the transition half-width of a single lipid usually was smaller than $1^{\circ}$, this error was negligible for these systems. It is small for lipid mixtures and lung surfactant. Relative temperature changes of $c_{p}$ maxima, induced by pressure, can be determined with a precision of about $0.001 \mathrm{~K}$. Differential densitometry was performed on two coupled DMA 602M cells (Anton Paar, Graz, Austria). One cell was filled with the sample and the other one with the reference buffer. Temperature control by a waterbath had an accuracy within 0.01-0.02 degrees. Combining two cells has the advantage that slight variations in environmental conditions (e.g., temperature drifts) take place simultaneously in both cells and do not show up in the difference of the responses of the two cells. This leads to an improvement of accuracy by about a factor of 20 . The instrument was calibrated with double distilled water and a saturated $\mathrm{NaCl}$ solution with known temperature dependence of the density. The data obtained in this experiment are the densities $\rho$ of the lipid dispersions, which are the inverse of the specific volume, $V$. The volume expansion coefficient, $\mathrm{d} V / \mathrm{d} T$, was derived from these data by fitting the temperature-dependent function $V(T)$ in a narrow window around the temperature $T$ with a polynomial. The volume expansion coefficient was then taken as the analytical derivative of the local fit function.

Extruded vesicles were made using a Lipofast extruder (Avestin Inc., Ottawa, Canada) using a polycarbonate filter with pore sizes of $100 \mathrm{~nm}$. The lung surfactant was washed such that it consisted only of the membrane fraction, i.e., the lipid fraction and the integral lung surfactant proteins SP-B and SPC. ${ }^{32,33}$

\section{Results}

3.1. Densitometry. We performed density measurements in our differential densitometer. In Figure 1 (top panel) the density profile of DMPC extruded vesicles (actually the difference between the density of the lipid dispersion and water) is shown. The density difference between a $10 \mathrm{mg} / \mathrm{mL}$ lipid dispersion and distilled water is given. The lipid density is larger than the density of water throughout the whole melting transition regime. A continuous decrease of the density with increasing temperature was observed, which displayed two pronounced steps. These data can be recalculated into the specific volume of the lipid (Figure 1, center panel). From these data the specific volume expansion coefficient can be deduced by local fitting and analytical derivative formation (bottom panel). It can be seen that the profile of the volume expansion coefficient displays two maxima. This is generally the case for extruded DMPC vesicles. $^{24}$

In the following we compare the volume expansion coefficient to the heat capacity of the identical vesicle preparations. The temperature dependence of the lipid volume shall be defined

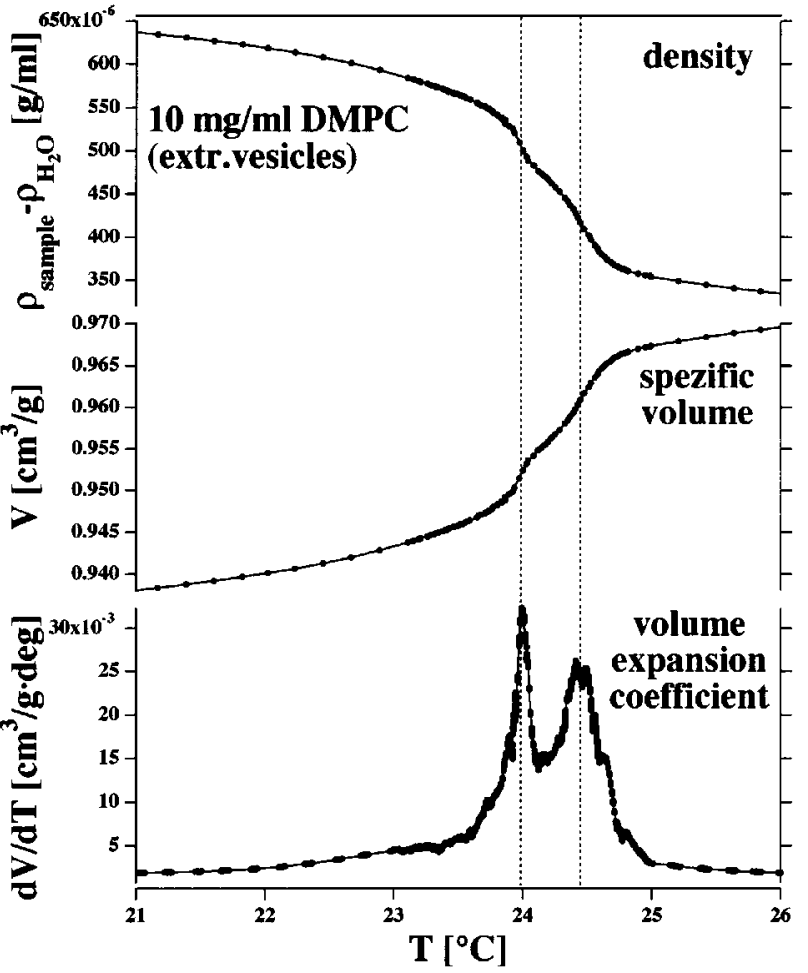

Figure 1. Densitometry data for extruded DMPC LUV. Top panel: difference of the density of a $10 \mathrm{mg} / \mathrm{mL}$ lipid dispersion and the water density, $\rho_{\text {sample }}(T)-\rho_{\mathrm{H}_{2} \mathrm{O}}(T)$. Middle panel: specific volume of the lipid, deduced from the data in the top panel. Bottom panel: specific volume expansion coefficient, $\mathrm{d} V(T) / \mathrm{d} T$ of extruded DMPC vesicles. The latter function has been obtained from the derivative of the specific volume profile.

as the sum of the intrinsic hydrocarbon chain volume, $V_{0}$, and the change in volume, $\Delta V$, induced by the cooperative melting transition:

$$
V(T)=V_{0}(T)+\Delta V(T), \quad \frac{\mathrm{d} V}{\mathrm{~d} T}=\text { const }+\frac{\mathrm{d}(\Delta V)}{\mathrm{d} T}
$$

Here we assumed that the temperature dependence of the intrinsic chain volume, $V_{0}$, is approximately linear with temperature in a small interval around the melting transition. This is justified from the constant offset of the $\mathrm{d} V / \mathrm{d} T$ data in Figure 2. Similarly, the temperature dependence of the enthalpy is given by

$$
\begin{aligned}
& H(T)=H_{0}(T)+\Delta H(T), \\
& \qquad \frac{\mathrm{d} H}{\mathrm{~d} T}=\mathrm{const}+\frac{\mathrm{d}(\Delta H)}{\mathrm{d} T}=c_{p, 0}+\Delta c_{p}
\end{aligned}
$$

where $\Delta H$ is the excess enthalpy change caused by the melting transition. The derivative of the enthalpy defines the heat capacity at constant pressure, $c_{p}$. In Figure 2 the volume expansion coefficient and the heat capacity are compared. We found a nearly exact proportional relationship, evident from the finding that the heat capacity and the volume expansion coefficient are nearly superimposable functions of the temperature. Thus,

$$
\frac{\mathrm{d}(\Delta V)}{\mathrm{d} T}=\gamma \cdot \Delta c_{p} \rightarrow \Delta V(T)=\gamma \cdot \Delta H(T)
$$

Here we introduced a constant factor, $\gamma$, which descibes the proportionality between excess enthalpy changes, $\Delta H$, and 


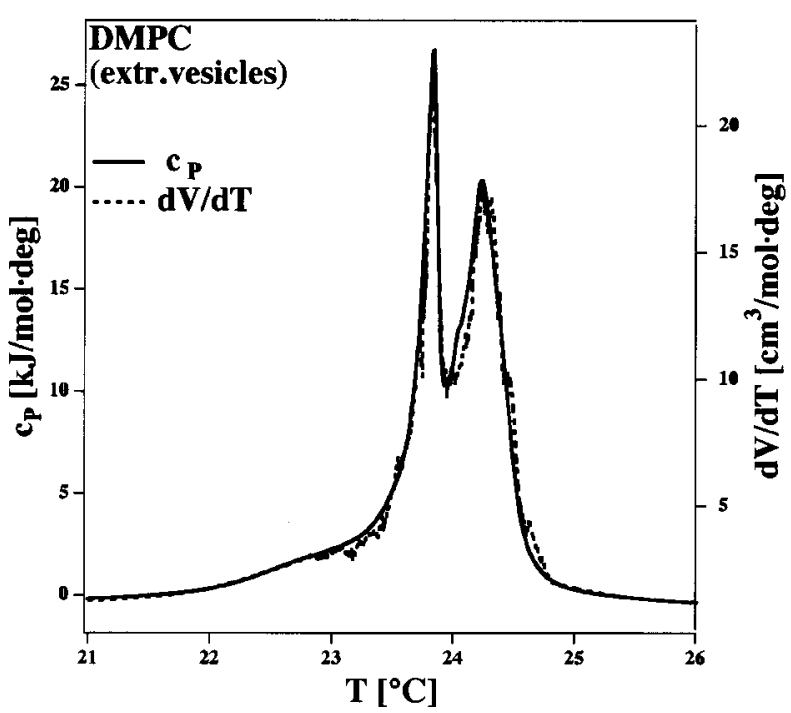

Figure 2. Heat capacity, $c_{p}$, and volume expansion coefficient, $\mathrm{d} V / \mathrm{d} T$ of extruded DMPC vesicles. The two curves are exactly superimposable, thus demonstrating the propotionality in the whole melting regime (cf. Table 1).

"excess volume" changes, $\Delta V$. The data in Figure 2 are quite remarkable because the proportional relationship between $\Delta c_{p}$ and $\mathrm{d}(\Delta V) / \mathrm{d} T$ is true even in minute details of the temperature profiles. We found this proportional relation to be true also for various lipid mixtures. In Figure 3 we show the proportional relationship between enthalpy and volume changes for DMPC: DPPC (left panel) and DLPC:DPPC mixtures (right panel). The proportional factors, $\gamma$, for these measurements have been obtained from visually superimposing the $c_{p}$ and the $\mathrm{d} V / \mathrm{d} T$ data and subsequent comparison of the scaling of the axes. The values obtained for different systems are summarized in Table. 1. For all systems studied, $\gamma$ was roughly the same (within experimental error) with an average of about $8.45 \times 10^{-4} \mathrm{~mL} / \mathrm{J}$. Errors have been determined from repeating experiments with several sample preparations of the same lipid.

Our density measurements are based on experiments in a vibrating capillary. ${ }^{34,35}$ The mean times for one period are recorded. The basic assumption when analyzing the data is that the lipid dispersion is homogeneous. Thus, inhomogeneous samples produce artifacts which result in incorrect quantitative numbers for the volume changes. Furthermore, to determine the proportional factor between enthalpy and volume changes, one has to determine the baseline of both density and calorimetric experiments and needs to know the exact concentration of the lipid. Thus, the experimental error for $\gamma$ is in the range of up to $10 \%$. For that reason, we now outline an alternative way to measure the proportional relation between volume and enthalpy changes, which is based on calorimetry with applied hydrostatic pressure. This method is shown to be much more accurate.

3.2. Pressure Calorimetry-Theory. In the following, pressure calorimetry is shown to be a very sensitive method to determine the temperature dependence of volume changes close to transitions. The aim of this study is to show that enthalpy and volume changes of lipids are proportional functions of the temperature. The mean enthalpy change of a given system at temperature $T$ and pressure $p_{0}$ with respect to a ground state is the weighted average over all possible states of the system:

$$
\langle\Delta H\rangle_{T}^{0}=\frac{\sum_{i} \Delta H_{i}^{0} \exp \left(-\Delta H_{i}^{0} / k_{\mathrm{B}} T\right)}{\sum_{i} \exp \left(-\Delta H_{i}^{0} / k_{\mathrm{B}} T\right)}
$$

The enthalpy of each microstate, $i$, at pressure $p_{0}$ is given by

$$
\Delta H_{i}^{0}=\Delta E_{i}+p_{0} \cdot \Delta V_{i}
$$

and, at a different pressure $p_{0}+\Delta p$, it is given by

$$
\Delta H_{i}^{\Delta p}=\Delta E_{i}+\left(p_{0}+\Delta p\right) \cdot \Delta V_{i}
$$

Let us now assume that the mean enthalpy change $\langle\Delta H\rangle$ is proportional to the mean volume change, $\langle\Delta V\rangle$. It has been shown that this is identical to that the relation between all $\Delta H_{i}$ and $\Delta V_{i}$ is a constant, ${ }^{24}$

$$
\langle\Delta H\rangle_{T}^{0}=\gamma \cdot\langle\Delta V\rangle_{\mathrm{T}} \Leftrightarrow \Delta H_{i}^{0}=\gamma \cdot \Delta V_{i}
$$

This relation has to be true if enthalpy and volume changes are proportional functions. Let us now assume that this is the case. It follows from eqs $5-7$ that

$$
\Delta H_{i}^{\Delta p}=\Delta E_{i}+p_{0} \cdot V_{i}+\gamma \cdot \Delta p \cdot \Delta H_{i}^{0}=\Delta H_{i}^{0} \cdot(1+\gamma \cdot \Delta p)
$$
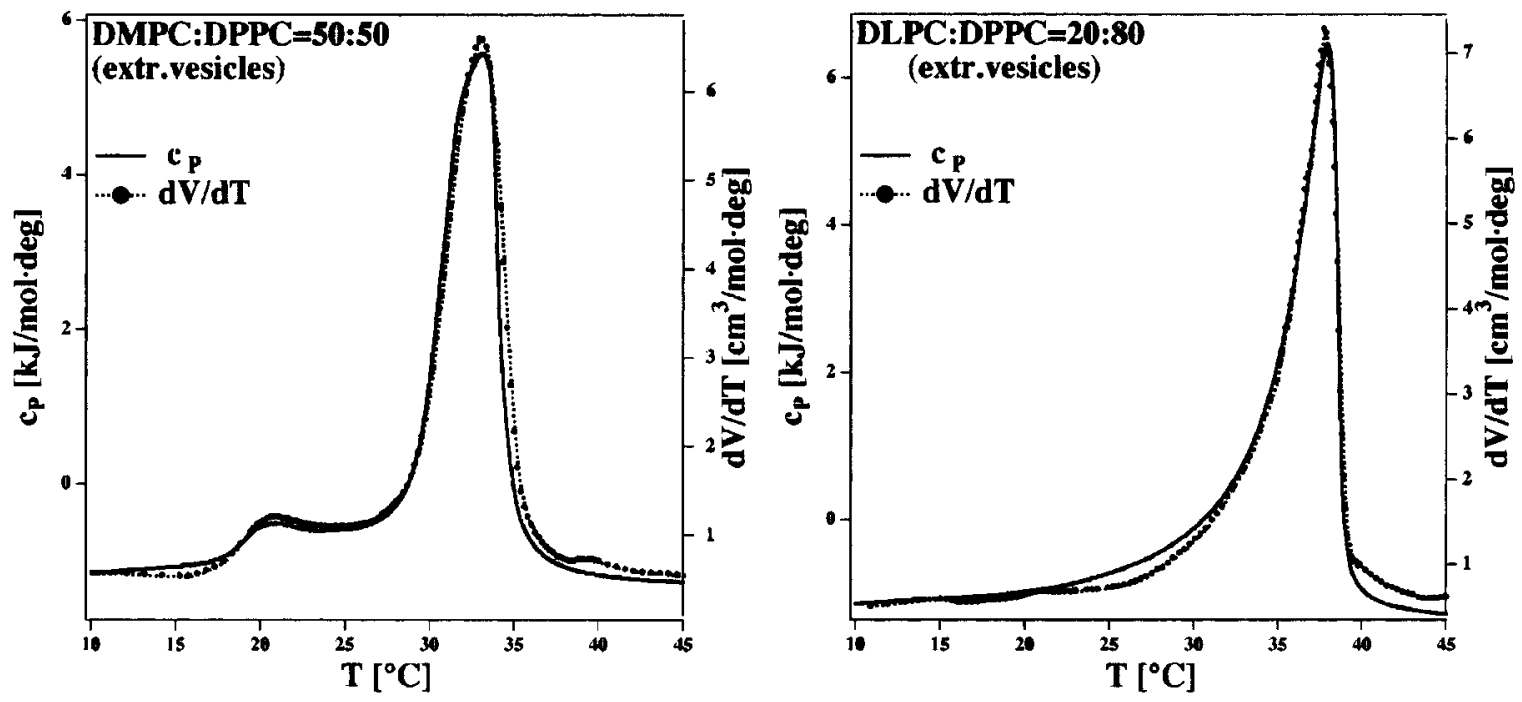

Figure 3. Heat capacity, $c_{p}$, and volume expansion coefficient, $\mathrm{d} V / \mathrm{d} T$ of two different lipid mixtures. Left: DMPC:DPPC $=50: 50 \mathrm{~mol} / \mathrm{mol}$, and Right: DLPC:DPPC $=20: 80 \mathrm{~mol} / \mathrm{mol}$. The two curves again follow near-proportional relations (cf. Table 1). 
Therefore the mean enthalpy at pressure $p_{0}+\Delta p$ is given by

$$
\begin{aligned}
\langle\Delta H\rangle_{T}^{\Delta p} & =(1+\gamma \cdot \Delta p) \frac{\sum_{i} \Delta H_{i}^{0} \exp \left(-(1+\gamma \cdot \Delta p) \Delta H_{i}^{0} / k_{\mathrm{B}} T\right)}{\sum_{i} \exp \left(-(1+\gamma \cdot \Delta p) \Delta H_{i}^{0} / k_{\mathrm{B}} T\right)} \\
& =(1+\gamma \cdot \Delta p) \frac{\sum_{i} \Delta H_{i}^{0} \exp \left(-\Delta H_{i}^{0} / k_{\mathrm{B}} T^{*}\right)}{\sum_{i} \exp \left(-\Delta H_{i}^{0} / k_{\mathrm{B}} T^{*}\right)}
\end{aligned}
$$

introducing a rescaled temperature $T^{*}$

$$
T^{*}=\frac{T}{1+\gamma \cdot \Delta p}
$$

From this it follows that the mean excess enthalpy at pressure $p_{0}+\Delta p$ and temperature $T$ is proportional to the enthalpy at pressure $p_{0}$ and temperature $T^{*}$ :

$$
\langle\Delta H\rangle_{T}^{\Delta p}=(1+\gamma \cdot \Delta p)\langle\Delta H\rangle_{T^{*}}^{0}
$$

This means that the excess heat capacity at pressures $p_{0}$ and $p_{0}+\Delta p$ are also closely related. After rescaling the temperature axis using eq 10 and dividing by $(1+\gamma \cdot \Delta p)$ the curves obtained at the two pressures have exactly the same shape. Relations 10 and 11 can therefore be used to determine the factor $\gamma$. Reversely, if the two curves have different shapes after rescaling, there is no exact proportional relation between enthalpy and volume changes. Below we are going to test the effect of pressure using pressure calorimetry.

3.3. Pressure Calorimetry-Experiments on Artificial Lipid Systems. The application of hydrostatic pressure leads to a shift in the melting profiles. This shift is dependent on the volume changes during the melting process. The accuracy of the determination of the volume change requires neither a knowledge about lipid concentration nor a determination of the baseline. Volume changes can be determined with the accuracy with which shifts in the calorimeter can be determined (provided that the applied pressure is constant). Pressure-induced shifts of melting profiles are shown in Figure 4. Let us first consider the left-hand panel (top traces). They show the heat capacity profile of extruded DMPC vesicles identical to that in Figure 1. Applied pressure (176 bar) leads to a shift of about $4 \mathrm{deg}$. The shape of the $c_{p}$ profile is maintained. Rescaling of the $c_{p}$

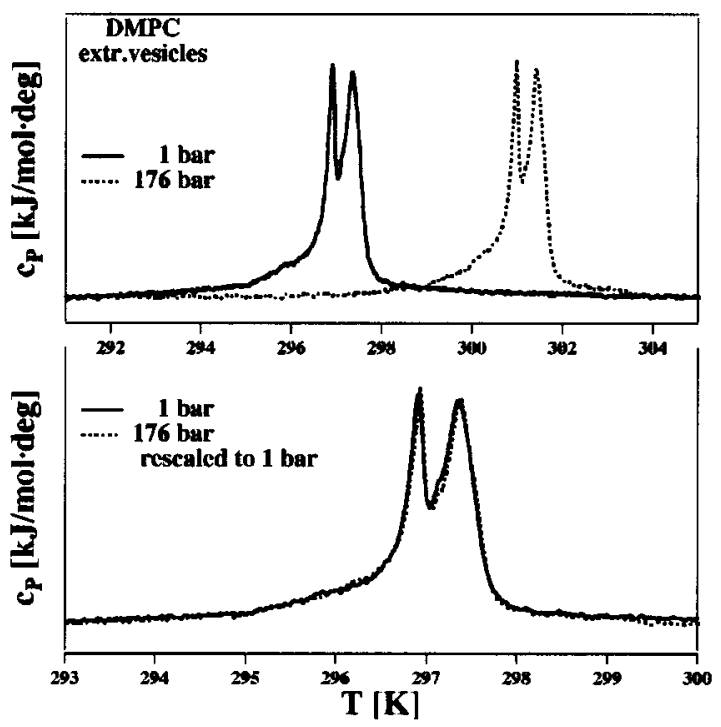

TABLE 1: Specific Volume Changes and $\gamma$-Factor Obtained from Densitometry

\begin{tabular}{llll}
\hline \multicolumn{1}{c}{ lipid system } & \multicolumn{1}{c}{$\begin{array}{c}\Delta H \\
{[\mathrm{~kJ} / \mathrm{mol}]}\end{array}$} & $\begin{array}{c}\Delta V \\
{\left[10^{-3} \mathrm{~mL} / \mathrm{j}\right]}\end{array}$ & $\begin{array}{c}\gamma \\
{\left[10^{-4} \mathrm{~mL} / \mathrm{g}\right]}\end{array}$ \\
\hline DMPC LUV extr. & $23.4 \pm 3.2$ & $30.7 \pm 5.2$ & $9.01 \pm 0.71$ \\
DPPC LUV extr. & $30.5 \pm 3.0$ & $35.2 \pm 7.5$ & $8.14 \pm 0.67$ \\
DMPC:DPPC = 50:50 extr. & 36.6 & 45.8 & 8.85 \\
DLPC:DPPC $=$ 20:80 extr. & 37.5 & 39.7 & 7.59 \\
& \multicolumn{3}{c}{ average $\bar{\gamma}=8.45 \pm 0.78$}
\end{tabular}

TABLE 2: $\gamma$-Factor Obtained from Pressure Calorimetry

\begin{tabular}{llllc}
\hline \multicolumn{1}{c}{ lipid system } & \multicolumn{1}{c}{$\begin{array}{c}T_{\mathrm{m}} \\
{\left[{ }^{\circ} \mathrm{C}\right]}\end{array}$} & $\begin{array}{c}\Delta p \\
{\left[10^{5} \mathrm{~Pa}\right]}\end{array}$ & $\begin{array}{c}\Delta T_{\max } \\
(\Delta p)\end{array}$ & $\begin{array}{c}\gamma^{\gamma} \\
{\left[10^{-4} \mathrm{~mL} / \mathrm{J}\right]}\end{array}$ \\
\hline DMPC LUV extr. & $23.77 ; 24.21$ & 175 & $4.02 ; 4.03$ & 7.761 \\
DMPC MLV & 23.56 & 167.5 & 3.85 & 7.841 \\
DPPC LUV extr. & 41.34 & 175 & 4.19 & 7.543 \\
DPPC MLV & 41.29 & 167.8 & 4.15 & 7.862 \\
DPPC MLV & 41.29 & 42 & 1.04 & 7.787 \\
DMPC:DPPC $=$ & 33.33 & 175 & 4.304 & 7.914 \\
$\quad$ 50:50 extr. & & & & 7.809 \\
bovine lung & 26.87 & 195 & 4.57 &
\end{tabular}

average $\bar{\gamma}=7.788 \pm 0.110$

profile obtained under pressure using eqs 10 and 11 leads to a nearly exact superposition of the $c_{p}$ profiles with and without pressure (bottom traces). According to the previous section this implies that the proportional relation between excess enthalpy and excess volume changes (eq 3 ) is nearly exactly true. In the right-hand panel of Figure 4 we display a similar experiment for extruded vesicles of DPPC. From the rescaling factor of the temperature axes one can calculate the proportional factor between enthalpy and volume changes. These values are listed in Table 2. For DMPC and DPPC they have equal values within error. Figure 5 displays similar experiments for multilamellar vesicles of DMPC and DPPC. These transitions display a very narrow transition half width and have to be performed at very slow scan rates $\left(0.2^{\circ} / \mathrm{h}\right)$. Although for such narrow transitions deviations from the strict proportionality should be most easily visible, the rescaling of the temperature axes leads to a nearly exact superposition of the curves with and without applied pressure.

Since DMPC and DPPC have identical values for $\gamma$, a 50:50 mixture of DMPC and DPPC displays a similar behavior (Figure 6 ). The heat capacity profile obtained under pressure can again

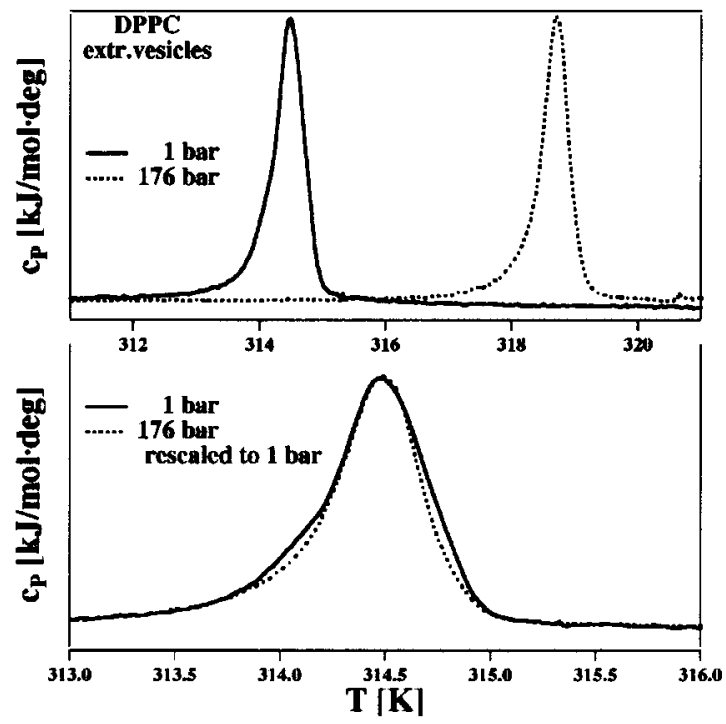

Figure 4. Heat capacity profiles of DMPC and DPPC unilamellar vesicles with and without excess bulk pressure. After rescaling of the temperature axes the two profiles are nearly exactly superimposable (cf. Table 2). 

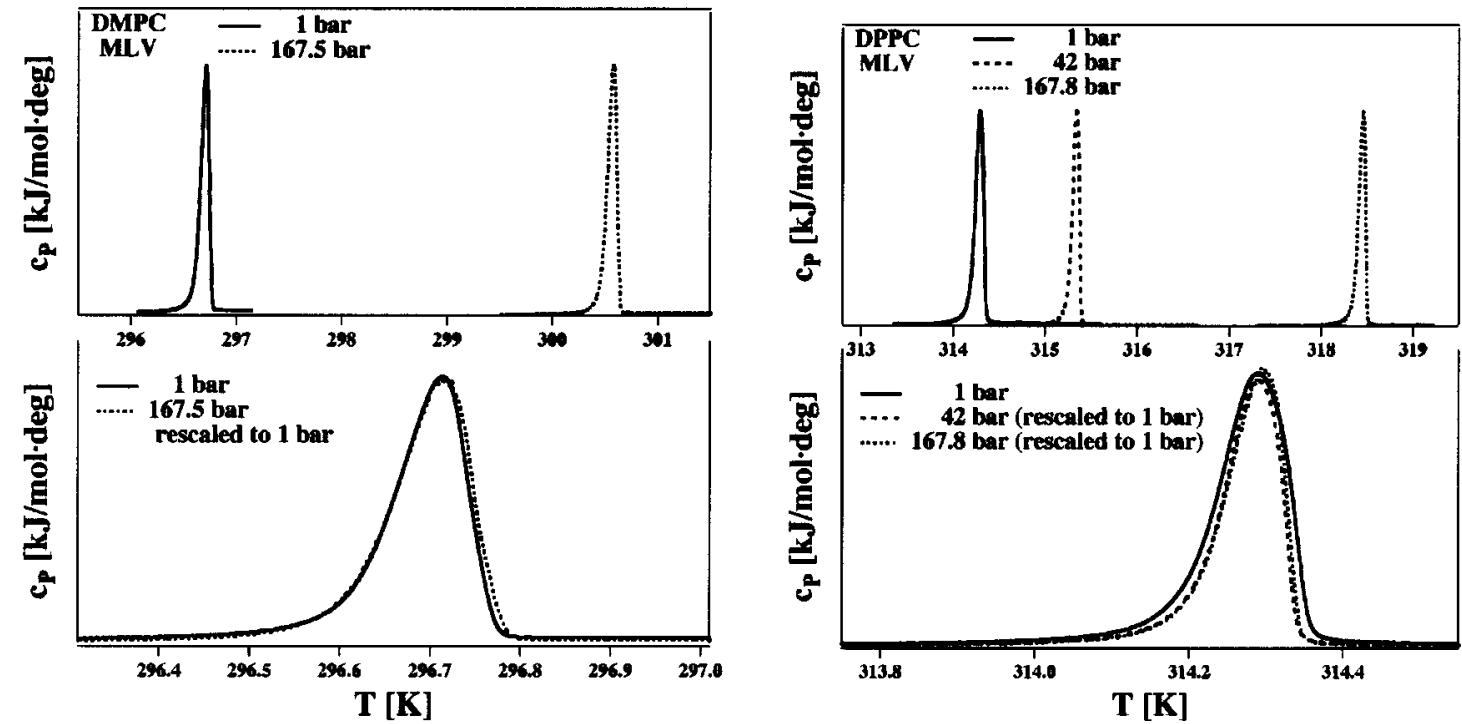

Figure 5. Heat capacity profiles of DMPC and DPPC multilamellar vesicles with and without excess bulk pressure. After rescaling of the temperature axes the two profiles are nearly exactly superimposable (cf. Table 2 ).

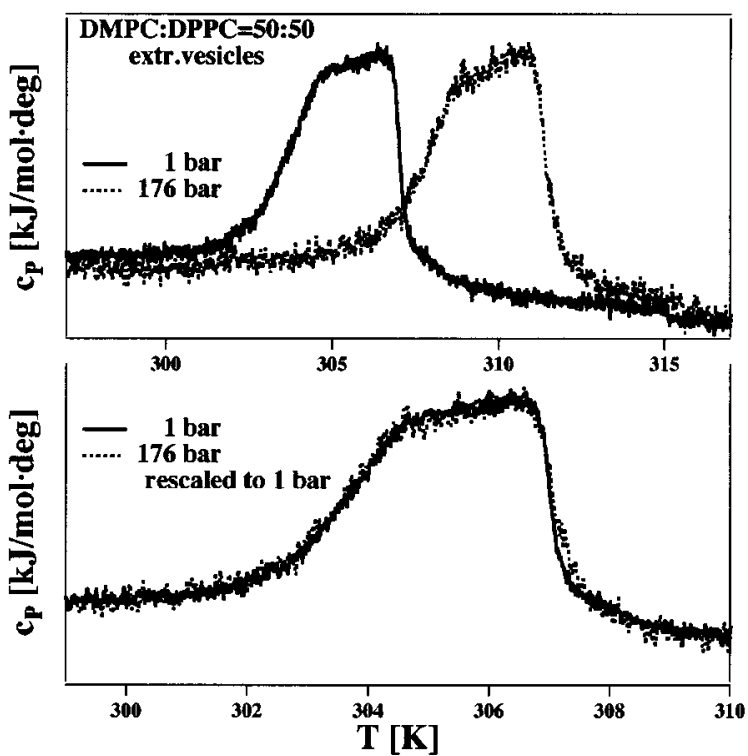

Figure 6. The pressure-induced shift of DMPC:DPPC $=50: 50 \mathrm{~mol} /$ mol extruded vesicles can perfectly be rescaled to the zero excess pressure profile. The scaling factor is the same as that for the pure lipids (see Table 2).

be superimposed with the profile obtained at athmospheric pressure by rescaling the temperature axes.

3.4. Biological Systems: Lung Surfactant. So far we have reported two interesting findings: (1) volume and enthalpy change in a proportional manner during the melting transition; (2) the proportionality constant within error is the same for the artificial membrane systems investigated. Therefore it would be interesting to know whether general mixtures of lipids display a similar behavior. For this reason we investigated melting processes in lung surfactant.

Lung surfactant is a biological system consisting of a mixture of lipids and of several surfactant proteins. ${ }^{32,33}$ Two of the surfactant proteins, SP-B and SP-C are membrane associated. The lung surfactant preparation used here has been washed such that it consists of lipids and the two integral membrane proteins. The lipid fraction of lung surfactant is heterogeneous with a high percentage (around 50\%) of DPPC. Heat capacity traces of lung surfactant are shown in Figure 7. It can be seen that
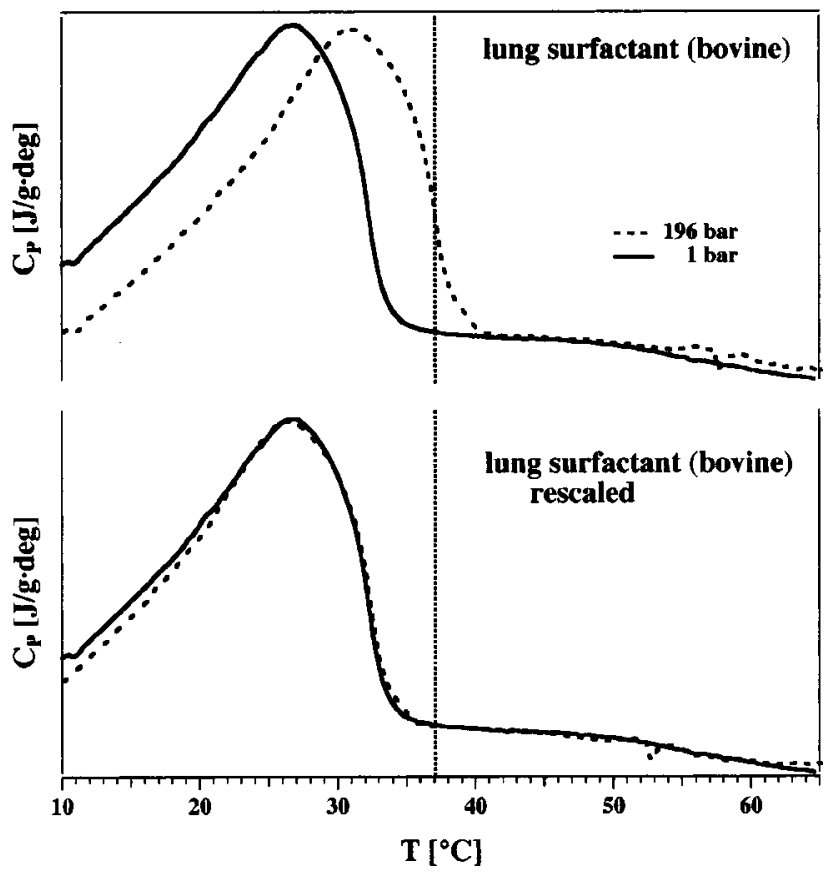

Figure 7. Bovine lung surfactant (containing the integral proteins SP-B and SP-C) displays a melting transition close to body temperature (indicated by the dotted line). The overall pressure-induced shift of the transition is nearly exactly the same as the shift in the model systems.

this biological sample displays a pronounced melting transition with a maximum at about $27^{\circ}$. The melting transition occurs over a broad temperature interval and ends slightly below body temperature. Application of 195 bar leads to a shift of the heat capacity maximum of about $4.6^{\circ}$. When multiplying the temperature axes with a constant factor one can superimpose the $c_{p}$ profile with and without pressure. Thus, even for this complex mixture, enthalpy and volume changes are proportional functions. More remarkably, the factor $\gamma$ within error is the same than for the artificial systems (Table 2).

3.5. Pretransition. Below the main transition the pretransition, a transition of small enthalpy is found. Between pretransition and main transition the surface structure of the membranes is altered and a periodic ripple pattern is formed. We have 

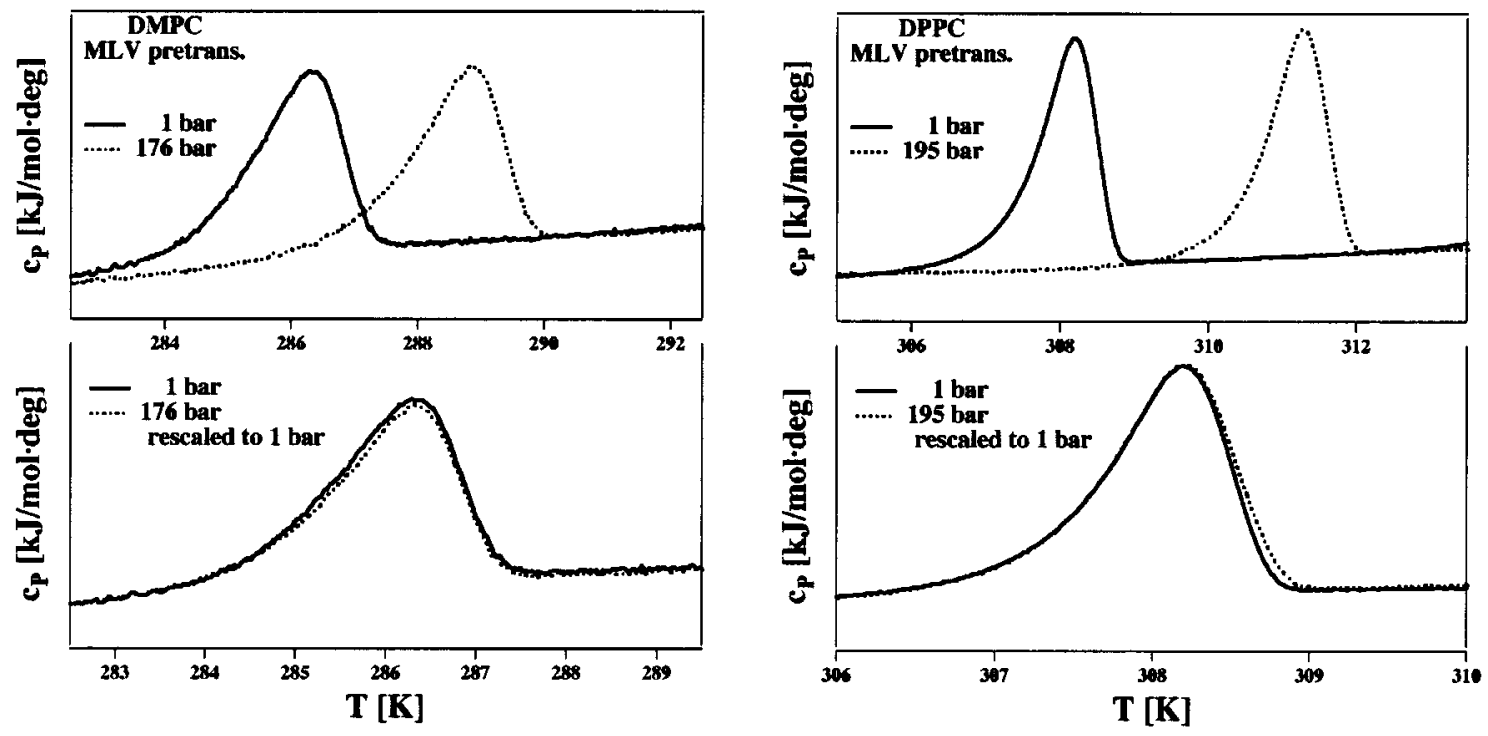

Figure 8. A proportional relation between enthalpy and volume changes is also obvious in the $c_{p}$-profiles of the pretransition. Interestingly, the overall shift is significantly smaller than the shift of the main transition, indicating a smaller overall volume change (see Table 3 ).

TABLE 3: $\gamma$-factor of the Pretransition Obtained from Pressure Calorimetry

\begin{tabular}{lcccc}
\hline $\begin{array}{l}\text { lipid system } \\
\text { pretransition }\end{array}$ & $\begin{array}{c}T_{\text {pre }} \\
{\left[{ }^{\circ} \mathrm{C}\right]}\end{array}$ & $\begin{array}{c}\Delta p \\
{\left[10^{5} \mathrm{~Pa}\right]}\end{array}$ & $\begin{array}{c}\Delta T_{\max } \\
(\Delta p)\end{array}$ & $\begin{array}{c}\gamma \\
{\left[10^{-4} \mathrm{~mL} / \mathrm{J}\right]}\end{array}$ \\
\hline DMPC MLV & 13.14 & 175 & 2.54 & 5.073 \\
DPPC MLV & 35.00 & 194 & 3.07 & 5.138 \\
& & & average $\bar{\gamma}=5.101 \pm 0.033$
\end{tabular}

recently proposed that the pretransition is part of the chain melting. ${ }^{31}$ The reason for the splitting of the chain melting process into two peaks is caused by a coupling to the structural change. If this was the case one would expect a pressure induced shift of the pretransition with a value comparable to the shift of the main transition. This is shown in Figure 8 for both the pretransition of multilamellar DMPC and DPPC vesicles. Interestingly, the pretransition is also shifted such that rescaling of the temperature axes leads to a superposition of the traces obtained with and without pressure. Thus, again enthalpy and volume changes are proportional functions. The proportionality constant, $\gamma$, is the same for both lipids (Table 3). However, its value is only about $65 \%$ of that for the main transition. This implies that the molecular processes in the pretransition and in the main transition are similar but not identical. Below we discuss the possibility that these differences are caused by the structural changes and related changes in the interaction with water.

\section{Discussion}

In this paper we have investigated the melting transition of artificial and biological lipid membranes with densitometry and calorimetry with and without pressure. The aim of our study is to demonstrate the high sensitivity of pressure calorimetry to measure volume changes in lipid membranes. We compared the temperature dependence of the volume to the enthalpy changes. Our main result is that to a very good approximation the two thermodynamic functions are proportional. The proportional constant was the same for all lipids investigated in this study, including complex biological mixtures. This finding is far from being trivial because it implies that this relation is true for all microstates of the canonical ensemble (cf. eq 8). The fact that the transition maximum shifts with applied pressure (which has been reported before) does not automatically imply that the shape of the transition is maintained. Actually, even slight deviations from an exact proportional relation between volume changes and enthalpy changes should have pronounced effects on the shape of the melting transition. The pressure dependence of the melting transition has been investigated before by various authors. ${ }^{12,13,16,18-21,36,37}$ It has been shown that the shift of the lipid melting transition is linear with the applied hydrostatic pressure in a pressure range up to at least 2 kbar. However, these authors were mainly interested in the shift of the transition point. This is reasonable as long as the volume and enthalpy changes at the transition are discontinuous. However, it has long been known that melting transitions have pronounced wings and may not be of first order. ${ }^{30,38}$ Therefore, both enthalpy and volume changes extend over a wide temperature regime. Thus, the purpose of this study is different from previous pressure studies on lipid phase transition insofar as here we are interested in the temperature-dependent shape of these transitions rather than in the absolute shift of transition point. For first-order transitions, which display an enthalpy discontinuity, the absolute temperature shift is sufficient to obtain numbers for volume changes. However, if the transitions are continuous, as they are for all lipid systems, the pressure dependence of the shape of the heat capacity profiles contains more information.

The pressure dependence of the melting profile of various lipids found by us $\left(\bar{\gamma}=7.79 \cdot 10^{-4} \mathrm{~mL} / \mathrm{J}\right.$, cf. Table 2) is comparable to previous studies for the transition temperature. The value for the proportionality factor, $\gamma$, averaged over various literature values yields a mean value $\bar{\gamma}=7.34 \cdot 10^{-4} \mathrm{~mL} / \mathrm{J}$ for DMPC MLV, $12,14,16,20,21 \quad \bar{\gamma}=7.07 \cdot 10^{-4} \mathrm{~mL} / \mathrm{J}$ for DPPC MLV, ${ }^{14,16,19-21,39,40}$ and $\bar{\gamma}=7.63 \cdot 10^{-4} \mathrm{~mL} / \mathrm{J}$ for distearoyl phosphatidylcholine MLV. ${ }^{13,20}$ Similar numbers where found for dieladioyl phoshatidylcholine and palmitoyl oleoyl phosphatidylcholine. Literature values for the pretransition $(\gamma=$ $4.56 \cdot 10^{-4} \mathrm{~mL} / \mathrm{J}$ for DMPC MLV, ${ }^{16}$ and $\gamma=5.26 \cdot 10^{-4} \mathrm{~mL} / \mathrm{J}$ for DPPC MLV $\left.{ }^{14}\right)$ are close to those found by us $\left(\gamma=5.10 \cdot 10^{-4}\right.$ $\mathrm{mL} / \mathrm{J}$, cf. Table 3).

There is also a considerable body of information available on density changes in the transition. Taking average values of enthalpy and volume changes from the literature, we arrive at 
values of $\bar{\gamma}=7.66 \cdot 10^{-4} \mathrm{~mL} / \mathrm{J}$ for DMPC MLV,2,6,41,42 and $\bar{\gamma}=7.39 \cdot 10^{-4} \mathrm{~mL} / \mathrm{J}$ for DPPC MLV., $, 5,7,8,10,11,41-45$

Thus, the general literature agrees that the pressure dependence of saturated phospholipids is very similar. Some unsaturated lipids with oleoyl chains seem to display a slightly lower pressure dependence. ${ }^{23}$ The finding of a proportional change of enthalpy and volume with temperature is of great relevance. Due to a well-known statistical thermodynamics theorem ${ }^{46}$ the heat capacity is proportional to the fluctuations in enthalpy

$$
c_{p}=\frac{\overline{H^{2}}-\bar{H}^{2}}{R T^{2}}
$$

and the isothermal compressibility $\left(\kappa_{T}=-(1 / V)(\mathrm{d} V / \mathrm{d} p)_{T}\right)$ is proportional to the fluctuations in volume

$$
\kappa_{T}=\frac{\overline{V^{2}}-\bar{V}^{2}}{\bar{V} \cdot R T}
$$

In a previous publication ${ }^{24}$ we showed that if $\Delta V(T)=\gamma \cdot \Delta H(T)$ for all temperatures around the transition, the fluctuations of volume and enthalpy are also proportional. Under these conditions one can directly translate the excess heat capacity into an "excess compressibility"

$$
\Delta \kappa_{T}(T)=\frac{\gamma^{2} \cdot T}{\bar{V}(T)} \cdot \Delta c_{p}
$$

This equation implies that heat capacity measurements are sufficient to predict isothermal compressibility changes. These predictions have been shown to be true in ultrasonic velocity measurements for DMPC/cholesterol systems. ${ }^{26} \mathrm{We}$ were also able to predict ultrasonic velocities for various other lipid mixtures (manuscript in preparation). A proportional relation between enthalpy and membrane area changes has also been suggested. ${ }^{24}$ If this was the case one can relate the heat capacity with the area compressibility and using a simple expression by Evans ${ }^{47}$ it can also be related to the bending elasticity. The predicted curvature elasticities ${ }^{24}$ and the experimentally obtained values $^{27,31}$ are in very good agreement, suggesting that the basic assumption on the proportional relation between area and enthalpy changes is correct.

Our findings suggest that heat capacity events can generally be related to changes in the elastic constants in a very simple manner. Since the proportionality constant between enthalpy and volume changes within error is the same for all lipids investigated here, it is possible to predict changes in the elastic constants from the heat capacity curves alone. This is true even for complex mixtures of unknown composition as lung surfactant. Lung surfactant is especially rich in DPPC but also contains various other lipids with different chain and headgroup composition as well as the surfactant proteins SP-B and SP-C.

It should be noted that a proportional relation between enthalpy and volume changes in transitions is not universal for all order transitions. In general, such a relation is not based on first principles. Therefore one has to take this finding as an experimental fact for lipid systems. The origin of this simple relationship in lipids is not exactly clear to us. One may argue that the predominant part of the enthalpy and volume changes in lipids are due to the internal degrees of freedom of the individual molecules. Thus, the change in overall enthalpy and volume is dominated by the sum of the contributions of all molecules rather than by nearest neighbor interactions and free volume between molecules. The melting of atomic crystals, on the other hand, is dominated by nearest neighbor interactions and changes of the free volume between atoms. In such a case no proportional relation between enthalpy and volume changes is expected. If this line of argument was correct one may expect that one can find similar proportional relationships for assemblies of other macromolecules, e.g., for the melting of waxes, fatty acids, and alkanes.

Finally, it should be added that we found lipid systems which do not obey the proportional relationship between volume and enthalpy changes. In all of these systems structural changes of the lipid matrix took place, which only occurred in the chain melting regime. ${ }^{29}$ The increased likelyhood of structural changes within the melting transition (which are linked to the change in the elastic constants) has been discussed above. Common to these "anomalous" systems is that the structural changes are related to changes in lipid hydration within the transition regime. We have recently proposed a model for the lipid pretransition, which implies that both, pretransition and main transition, are related to chain melting. If this were the case one may wonder why the value for $\gamma$ is different for the pretransition and the main transition. However, the formation of a periodic ripple pattern on the surface is accompanied by changes in headgroup hydration. ${ }^{31}$ Thus, we assume that the value for the pretransition is smaller because it contains contributions from the hydration layer of the ripple phase. Therefore, it seems to be likely that pressure-induced changes in hydration can also be seen in the heat capacity traces and in principle be separated from the changes in the lipid chain volume. A manuscript on heat capacities and pressure dependence of these "anomalous" lipid systems is in preparation.

\section{Conclusions}

We show that pressure calorimetry can serve as a valuable tool to measure the proportional relation between volume and enthalpy changes very accurately. Combined with densitometry we have shown that for all systems investigated the melting transition follows a nearly exact proportional relationship between volume and enthalpy changes, and that the proportionality constant is identical for all systems. This is true even for biological systems. Thus, these data provide an important basis for relating model studies to complex systems of unknown composition, especially when linking the heat capacity to the elastic constants of the membrane.

Acknowledgment. We are grateful to Fred Possmeyer for useful discussion and the donation of a lung surfactant preparation. This work has been made possible by grants from the "Studienstiftung des deutschen Volkes" and from the "Deutsche Forschungsgemeinschaft" (He1829/6-1).

\section{References and Notes}

(1) Cevc, G.; Marsh, D. Phospholipid Bilayers: Physical Principles and Models; Wiley: New York, 1989.

(2) Nagle, J. F.; Wilkinson, D. A. Biophys. J. 1978, 23, 159-175.

(3) Marsh, D. CRC Handbook of Lipid Bilayers; CRC Press: Boca Raton, FL, 1990.

(4) Nagle, J. F. Biophys. J. 1993, 64, 1476-1481.

(5) Blazyk, J. F.; Melchior, D. L.; Steim, J. M. Anal. Biochem. 1975, $68,586-599$.

(6) Epand, R. M.; Epand, R. F. Chem. Phys. Lett. 1980, 27, 139-150.

(7) Wilkinson, D. A.; Nagle, J. F. Anal. Biochem. 1978, 20, 263-271.

(8) Melchior, D. L.; Scavitto, F. J.; Steim, J. M. Biochemistry 1980, 19, 4828-4834.

(9) Wilkinson, D. A.; Nagle, J. F. Biochemistry 1981, 20, 187-192.

(10) Anthony, F. H.; Biltonen, R. L.; Freire, E. Anal. Biochem. 1981, $116,161-167$. 
(11) Wiener, M. C.; Tristam-Nagle, S.; Wilkinson, D. A.; Campell, L. E.; Nagle, J. F. Biochim. Biophys. Acta 1988, 938, 135-142.

(12) Böttner, M.; Ceh, D.; Jacobs, U.; Winter, R. Z. Phys. Chem. 1994, $184,205-218$.

(13) Maruyama, S.; Matsuki, H.; Ichimori, H.; Kaneshina, S. Chem. Phys. Lett. 1996, 82, 125-132.

(14) Ichimori, H.; Hata, T.; Yoshioka, T.; Matsuki, H.; Kaneshina, S. Chem. Phys. Lett. 1997, 89, 97-105.

(15) Ichimori, H.; Hata, T.; Matsuki, H.; Kaneshina, S. Biochim. Biophys. Acta 1998, 1414, 165-174.

(16) Wong, P. T. T.; Siminovitch, D. J.; Mantsch, H. H. Biochim. Biophys. Acta 1988, 947, 139-171.

(17) Bernsdorff, C.; Wolf, A.; Winter, R. Z. Phys. Chem. 1996, 193 $151-173$.

(18) Czeslik, C.; Reis, O.; Winter, R.; Rapp, G. Chem. Phys. Lett. 1998

91, 135-144.

(19) Utoh, S.; Takemura, T. Jpn. J. Appl. Phys. 1985, 24, 356-360.

(20) Braganza, L. F.; Worcester, D. L. Biochemistry 1986, 25, 25912596.

(21) Winter, R.; Pilgrim, W.-C. Ber. Bunsen-Ges. Phys. Chem. 1989, 93, 708-717.

(22) Czeslik, C.; Winter, R.; Rapp, G.; Bartels, K. Biophys. J. 1995, $68,1423-1429$

(23) Winter, R.; Gabke, A.; Erbes, J.; Czeslik, C. In High-pressure molecular science; Winter, R., Jonas, J., Eds.; Kluwer Academic Publishers: Norwell, MA, 1999; pp 369-403.

(24) Heimburg, T. Biochim. Biophys. Acta 1998, 1415, 147-162.

(25) Mitaku, S.; Ikegami, A.; Sakanishi, A. Biophys. Chem. 1978, 8, 295-304.

(26) Halstenberg, S.; Heimburg, T.; Hianik, T.; Kaatze, U.; Krivanek, R. Biophys. J. 1998, 75, 264-271. 356.

(27) Dimova, R.; Pouligny, B.; Dietrich, C. Biophys. J. 2000, 79, 340-

(28) Heimburg, T. Curr. Opin. Coll. Interface Sci. 2000, 5, 224-231.

(29) Schneider, M. F.; Marsh, D.; Jahn, W.; Kloesgen, B.; Heimburg,

T. Proc. Natl. Acad. Sci. U.S.A. 1999, 96, 14312-14317.
(30) A histogram method to obtain heat capacities in lipid monolayers curved bilayers and membranes containing peptides. Ivanova, V. P.; Heimburg, T.

(31) Heimburg, T. Biophys. J. 2000, 78, 1154-1165.

(32) Yu, S.; Harding, P. G.; Smith, N.; Possmeyer, F. Lipids 1983, 18, $522-529$.

(33) Veldhuizen, R.; Nag, K.; Orgeig, S.; Possmeyer, F. Biochim. Biophys. Acta 1998, 1408, 90-108.

(34) Kratky, O.; Leopold, H.; Stabinger, H. Z. Angew. Phys. 1969, 27, $273-277$.

(35) Kratky, O.; Leopold, H.; Stabinger, H. Methods Enzymol. 1973, $27,98-110$ 4.

(37) Landwehr, A.; Winter, R. Ber. Bunsen-Ges. Phys. Chem. 1994, 98 $1585-1589$.

(38) Mouritsen, O. G.; Boothroyd, A.; Harris, R.; Jan, N.; Lookman, T.; MacDonald, L.; Pink, D. A.; Zuckermann, M. J. J. Chem. Phys. 1983, 79, 2027-2041.

(39) Liu, N.-I.; Kay, R. L. Biochemistry 1977, 16, 3484-3486.

(40) Mountcastle, D. B.; Biltonen, R. L.; Halsey, M. J. Proc. Natl. Acad. Sci. U.S.A. 1978, 75, 4906-4910.

(41) Janiak, M. J.; Small, D. M.; Shipley, G. G. Biochemistry 1976, 15, 4575-4580.

(42) Mabrey, S.; Sturtevant, J. M. In Methods in Membrane Biology, Vol. 9; Korn, E., Ed.; Plenum Press: New York, 1978; pp 237-274.

(43) Posch, M.; Rakusch, U.; Mollay, C.; Laggner, P. J. Biol. Chem. 1983, 258, 1761-1766.

(44) MacDonald, R. C. Biochim. Biophys. Acta 1978, 507, 26-37.

(45) Ohki, K.; Tamura, K.; Hatta, I. Biochim. Biophys. Acta 1990, 1028, $215-222$

(46) Hill, T. L. An introduction to statistical thermodynamics; Dover Publications: New York, 1960.

(47) Evans, E. A. Biophys. J. 1974, 14, 923-931. 